\title{
GLOBAL EXISTENCE AND ASYMPTOTIC BEHAVIOR OF A MODEL FOR BIOLOGICAL CONTROL OF INVASIVE SPECIES VIA SUPERMALE INTRODUCTION*
}

\author{
RANA D. PARSHAD ${ }^{\dagger}$, SAID KOUACHI ${ }^{\ddagger}$, AND JUAN B. GUTIERREZ ${ }^{\S}$
}

\begin{abstract}
The purpose of this manuscript is to propose a model for the biological control of invasive species, via introduction of phenotypically modified organisms into a target population. We are inspired by the earlier Trojan Y Chromosome model [J.B. Gutierrez, J.L. Teem, J. Theo. Bio., 241(22), 333-341, 2006]. However, in the current work, we remove the assumption of logistic growth rate, and do not consider the addition of sex-reversed supermales. Also the constant birth and death coefficients, considered earlier, are replaced by functionally dependent ones. In this case the nonlinearities present serious difficulties since they change sign, and the components of the solution are not a priori bounded, in some $L^{p}$-space for $p$ large, to permit the application of the well known regularizing effect principle. Thus functional methods to deduce the global existence in time, for the system in question, are not applicable. Our techniques are based on the Lyapunov functional method. We prove global existence of solutions, as well as existence of a finite dimensional global attractor, that supports states of extinction. Our analytical finding are in accordance with numerical simulations, which we also present.
\end{abstract}

Key words. Reaction-diffusion system, global existence, Lyapunov functional, global attractor, invasive species management.

AMS subject classifications. 35B40, 35B41, 35Q92, 37B25, 92D25.

\section{Introduction}

Exotic species, commonly referred to as invasive species, are in some instances capable of establishing a self-sustained population when they propagate to new environments. Once established, they can be extremely difficult to eradicate, or even manage $[10,41]$. There are numerous cases of harm to the environment and the economy due to the action of various invasive species [30, 29]. Some well known examples of these species include the Burmese python in southern regions of the United States, the cane toad in Australia, and the sea lamprey and round goby in the Great Lakes region in the northern United States [8]. The cane toad was brought into Australia from Hawaii in 1935, to control the cane beetle. They multiplied rapidly since their introduction, and have negatively affected biodiversity in the region [38]. The sea lamprey entered the great lakes in the 1800s through shipping canals. Their population has since exploded [4], and these predators have caused a severe decline in lake trout and other lake fish populations, adversely affecting various local fisheries. The Burmese python population has been exponentially on the rise in the Florida glades since the 1980s. This has been attributed to a number of reasons, such as the exotic pet trade in Florida. Climatic factors might support their spread to a third of the United States [32]. Invasive species, aside from being considered one of the greatest threats to biodiversity, cause agricultural losses worldwide in the range of several

*Received: May 15, 2012; accepted (in revised form): October 17, 2012. Communicated by Paul Milewski.

${ }^{\dagger}$ Mathematics and Computer Science and Engineering Division, King Abdullah University of Science and Technology, Thuwal 23955-6900, Kingdom of Saudi Arabia (rana.parshad@kaust.edu.sa).

${ }^{\ddagger}$ Department of Mathematics, College of Science, Qassim University, P.O. Box 6644, Al-Gassim, Buraydah 51452, Kingdom of Saudi Arabia (kouachi@hotmail.com).

$\S$ Department of Mathematics \& Institute of Bioinformatics, University of Georgia, Athens, GA 30602, USA (jgutierr@uga.edu). 
hundred billion dollars [8]. To date, there have been no completely effective methods for eradicating or containing these species in real scenarios, in the wild.

It has been observed that the size of a population can be affected as a result of shifting the sex ratio [12]; for example, by the introduction of large quantities of sterile males. This approach has the advantage of targeting only the invasive species. Even though this strategy has been successfully applied toward the control of fish such as the sea lamprey in the Great Lakes [45], it has been more commonly used in the control of insects. The release of large numbers of sterile male fruit flies in Florida reduces the probability that a fertile female will encounter a fertile male and produce viable progeny. Because male fruit flies do not themselves cause crop damage, an overwhelming number of sterile males can be released into the environment to compete with fertile males in matings [20]. If insufficient numbers of sterile males are released, normal matings may occur in sufficient numbers to maintain the population.

Although changing the sex ratio of a population seems difficult to achieve, a precedent for reducing a fish population as a result of altered sex ratios through sex reversal exists in a study involving Chinook salmon in the Columbia River [28]. The study involved genotype analysis of 50 males and 50 females removed from the river. DNA from each fish was analyzed for the presence of Y-chromosome specific DNA using a DNA probe. The study revealed that the 50 males were genotypic XY males $(m)$ as expected. However, the majority of females also tested positive for the Ychromosome DNA sequence, indicating that they were actually genotypic XY males that had become feminized for an unknown reason, possibly as a result of exposure to estrogen-like chemicals in the environment [31]. The study highlighted the likely impact of XY females $\left(f_{x y}\right)$ on the population, which was a long-term reduction in normal XX females $(f)$. This reduction would take place because of a reduced number of females occurring in progeny resulting from $f_{x y}$ mating with $m$, and also from the effects of YY males ( $s$, also called supermales) produced from these matings. This $s$ genotype would be expected to produce only males as progeny resulting from their matings with $f$, as shown in figure 1.1. The expected result of having $f_{x y}$ is a future decline of the population due to a reduction in normal $f$. Although this example involves an indigenous species rather than an exotic one, the same principle is potentially useful as a tool to reduce nonindigenous fish populations in the wild.
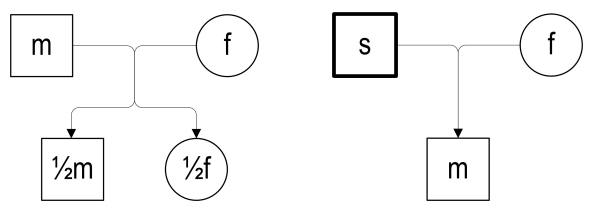

FIG. 1.1. The left figure shows mating of a $(X X)$ female $(f)$ and a $(X Y)$ male $(m)$; the progeny of this cross produces half females and half males. The right figure shows mating of a $(X X)$ female $(f)$ and a $(Y Y)$ supermale $(s)$; this cross always results in male progeny.

It has also been reported that environmental pressure for masculinization would lead to extinction of fish populations with a XY sex-determination system [18]. Masculinization by exposure to androgens has been reported in the wild [19], and is a common practice in salmonid industries to produce all-female stocks [17]. The case of environmental pressure to feminize, in theory at least, produces individuals that are $\mathrm{YY}$ and thus the population contains $s$.

A number of models have considered control of invasive species through the ad- 
dition of engineered individuals that drive the population to local extinction. Among these strategies, the Daughterless Carp [3] and the Trojan Sex Chromosome [9, 35, 36], have been particularly well studied. The first strategy aims to causing extinction via the release of individuals with insertions of aromatase inhibitor genes. The latter strategy tries to cause extinction via the constant release of sex-reversed supermales, denoted $r$ in (1.4), into a target population. Both strategies have the difficulty of requiring sophisticated biological techniques of genetic manipulation, and phenotypical identification. We briefly recount the TYC model [35]

$$
\begin{aligned}
\partial_{t} f & =D \Delta f+\frac{1}{2} f m \beta L-\delta f \\
\partial_{t} m & =D \Delta m+\left(\frac{1}{2} f m+\frac{1}{2} r m+f s\right) \beta L-\delta m \\
\partial_{t} s & =D \Delta s+\left(\frac{1}{2} r m+r s\right) \beta L-\delta s \\
\partial_{t} r & =D \Delta r+\mu-\delta r
\end{aligned}
$$

with the boundary conditions

$$
f=m=s=r=0 \quad \text { on } \mathbb{R}^{+} \times \partial \Omega .
$$

Here $\Omega \subset R^{3}$ is a bounded domain. Also $L=1-\left(\frac{f+m+r+s}{K}\right)$, where $K$ is the carrying capacity of the ecosystem, $D$ is a diffusivity coefficient, $\beta$ is a birth coefficient and $\delta$ is a death coefficient. We prescribe suitable initial data. Notice, due to the presence of the logistic control term, the components are a priori uniformly bounded, and we have a maximum principle, or $L^{\infty}$ control on the individual components $f, m, r$, and $s$ of the system [36]. The results derived in $[9,35,36]$ are done so via the use of this estimate, coupled with standard energy methods.

In the current manuscript we study a model for biological control, where we remove the logistic type term, present in the prior model. We also assume nonlinear and functionally dependent birth and death rates, instead of the constant coefficient birth and death rates assumed earlier. In this case the current system poses serious mathematical difficulties, as the nonlinearities change sign, and neither the $f$ nor $m$ component of the solution is a priori bounded in some $L^{p}$ space. The eradication strategy works as follows. A genetically modified individual, denoted $s(\mathrm{YY})$, bearing two $\mathrm{Y}$ chromosomes, is introduced at a rate $\mu(s)$ to a target population of an invasive species containing normal females and males, denoted as $f$ and $m$ respectively. Mating between the introduced $s$ and the invasive female $f$, always leads to a male progeny $m$. This generates a disproportionate number of males over time. The higher incidence of males decrease the female to male ratio. Ultimately, the number of $f$ decline to zero, causing local extinction. This strategy is relevant to organisms with an XY sexdetermination system, in which males carry one $\mathrm{X}$ chromosome and one $\mathrm{Y}$ chromosome $(\mathrm{XY})$, and females carry two $\mathrm{X}$ chromosomes (XX). The primary purpose of the current manuscript is to show that extinction is always possible, via the proposed strategy, even in a population which is not governed by a logistic type control term.

The control method is modeled via the following system of reaction diffusion equations:

$$
\begin{aligned}
\partial_{t} f & =d_{1} \Delta f+g_{1}(f, m, s) f m-g_{2}(f, m, s) f, \\
\partial_{t} m & =d_{2} \Delta m+g_{3}(f, m, s) f m+g_{4}(f, m, s) f s-g_{5}(f, m, s) m,
\end{aligned}
$$




$$
\partial_{t} s=d_{3} \Delta s+\mu(s)-g_{6}(s) s
$$

in $\mathbb{R}^{+} \times \Omega$, with the boundary conditions

$$
\partial_{\eta} f=\partial_{\eta} m=\partial_{\eta} s=0, \quad \text { on } \mathbb{R}^{+} \times \partial \Omega,
$$

where $\Omega$ is an open bounded domain in $\mathbb{R}^{N}$ with smooth boundary $\partial \Omega$, and $\partial_{\eta}$ denotes the outward normal derivative on $\partial \Omega$. Note that the results we have derived in the current manuscript, for global existence and global attractor, are valid even for Dirichlet boundary conditions. The choice of boundary condition depends on the application at hand. The Neumann boundary condition is used more often, as the Dirichlet boundary condition is left to applications where there is annihilation/death of species on the boundary. It could be used if we were implementing the proposed strategy in a physical domain where a poison such as rotenone (actively used as a toxic control in fisheries), has been sprayed around the boundary, forcing the species that reach the boundary to die.

$d_{1}, d_{2}$, and $d_{3}$ are the diffusion coefficients. The initial data

$$
f(0, x)=f_{0}(x), m(0, x)=m_{0}(x), s(0, x)=s_{0}(x) \text { in } \Omega
$$

are assumed to be nonnegative and uniformly bounded on $\Omega$.

The mating rates $g_{1}, g_{3}$, and $g_{4}$, and death rates $g_{2}$ and $g_{5}$ are assumed nonlinear, and have polynomial growth.

Equation (1.8) is independent of the two first equations. It is the heat equation under homogeneous Neumann boundary conditions. Under standard conditions on the reaction term $r_{3}$ :

$$
\frac{\partial^{2}\left[\mu(s)-g_{6}(s) s\right]}{\partial s^{2}} \leq 0 \text { and } \int_{\max _{x \in \bar{\Omega}} s_{0}(x)}^{+\infty} \frac{d s}{\mu(s)-g_{6}(s) s}=+\infty
$$

see [5].

The solution of (1.8) with the given boundary conditions exists globally in time and is bounded on $\Omega$,

$$
\|s(t, .)\|_{\infty} \leq s_{\infty}(t) \text { in } \mathbb{R}^{+},
$$

where $s_{\infty}$ is a bounded function on bounded subsets of $\mathbb{R}^{+}$.

As aforementioned the mathematical difficulty in analyzing (1.6)-(1.8) is that the reaction terms do not have a constant sign. This means that none of the equations are good in the sense that neither $f$ nor $m$ is a priori bounded or at least bounded in some $L^{p}$-space for $p$ large, so that one cannot apply the well known regularizing effect.

In the case when the nonlinearities have a constant sign many results have been obtained: When $r_{1}(f, v)=-f m^{\beta}$ (which implies the uniform boundedness of $f$ ) and $r_{2}(f, m)=f m^{\beta}, \mathrm{N}$. Alikakos [2] established global existence and $L^{\infty}$-bounds of positive solutions when $1<\beta<\frac{(n+2)}{n}$. K. Masuda [33] showed that the solutions exist globally for every $\beta>1$. S. L. Hollis, R. H. Martin and M. Pierre [16] established global existence of positive solutions for system (1.6)-(1.7) with the boundary conditions

$$
\lambda_{1} f+\left(1-\lambda_{1}\right) \frac{\partial f}{\partial \eta}=\beta_{1}, \lambda_{2} m+\left(1-\lambda_{2}\right) \frac{\partial m}{\partial \eta}=\beta_{2} \quad \text { on } \mathbb{R}^{+} \times \partial \Omega
$$


where

$$
0<\lambda_{1}, \lambda_{2}<1, \lambda_{1}=\lambda_{2}=1, \beta_{1} \geq 0 \text {, and } \beta_{2} \geq 0 \text {, or } \lambda_{1}=\lambda_{2}=\beta_{1}=\beta_{2}=0 \text {, }
$$

under the conditions of the uniform boundedness of $f$ on $\left[0, T_{\max }\right] \times \Omega$, and

$$
r_{1}(u, v)+r_{2}(u, v) \leq C(u, v)(u+v+1), \text { for all } u \geq 0 \text { and } v \geq 0,
$$

with $r_{1}$ and $r_{2}$ can change sign and where $C(u, v)$ is positive and uniformly bounded function defined on $\mathbb{R}^{+} \times \mathbb{R}^{+}$. Haraux and A. Youkana [13] simplified the proof of $\mathrm{K}$. Masuda [33], while using techniques based on Lyapunov functionals and while taking nonlinearities $r_{1}(f, m)=-r_{1}(f, m)=-f R(m)$ satisfying the condition

$$
\lim _{v \rightarrow+\infty}\left[\frac{\log (1+R(v))}{v}\right]=0,
$$

where $R(v) \geq 0$ for all $v \geq 0$. S. Kouachi and A. Youkana [24] generalized the results of A. Haraux and A. Youkana [13] while adding $-c \Delta f$ to the right-hand side of the second equation of system (1.6)-(1.9) and the condition

$$
\lim _{v \rightarrow+\infty}\left[\frac{\log \left(1+r_{1}(u, v)\right)}{v}\right]<\alpha^{*}, \text { for any } u \geq 0,
$$

where $R(u, v) \geq 0$ for all $u, v \geq 0$ and

$$
\alpha^{*}=\frac{2 d_{1} d_{2}}{n\left(d_{1}-d_{2}\right)^{2}\left\|f_{0}\right\|_{\infty}} .
$$

This condition reflects the weak exponential growth of the reaction term $f$. One notices that condition (1.15) is insufficient to prove global existence for solutions to system (1.6)-(1.9) and authors usually impose that $r_{1}$ (or $r_{2}$ ) also satisfies the following analogous condition:

$$
r_{1}(u, v)\left(\text { or } r_{2}(u, v)\right) \leq C^{\prime}(u, v)(u+v+1) \text {, for all } u \geq 0 \text { and } v \geq 0 .
$$

In the case when the nonlinearities do not have a constant sign, there are not many results in the literature; A. J. Morgan [34] generalized the results of [16] to show that solutions of the m-components reaction diffusion systems exist globally $(m \geq 2)$ where also, in our case $(m=2)$, he imposed condition (1.15) on $f$ and $f+g$ under the boundary conditions (1.16). S. L. Hollis [15] extended the results, under the same conditions, to the boundary conditions (1.16), but he took

$$
0 \leq \lambda_{1}, \lambda_{2} \leq 1, \beta_{1} \geq 0 \text { and } \beta_{2} \geq 0 .
$$

See [11] for related results. In $[24,27,1]$, we generalized the above results respectively for two, three, and finally $m$ components systems:

$$
\frac{\partial f_{i}}{\partial t}-d_{i} \Delta f_{i}=r_{i}\left(f_{1}, \ldots, f_{m}\right) \quad \text { in } \mathbb{R}^{+} \times \Omega ; i=1, \ldots, m,
$$

under the unique condition

$$
\sum_{i=1}^{m} D_{i} r_{i}\left(f_{1}, \ldots, f_{m}\right) \leq C\left[1+\sum_{i=1}^{m} f_{i}\right]
$$


for all positive constants $D_{i}$ sufficiently large, where $C$ is positive constant and we showed the global existence without imposing the boundedness of one of the components of the solution.

It is usual that under the action of diffusion, dynamical systems tend to smooth out. However, an inherent difficulty in the system (1.6)-(1.9) is that the asymptotic sign condition in vector version

$$
\limsup _{|s| \rightarrow \infty} F(s) \cdot s \leq C
$$

(where $C$ is a positive constant and $F$ is the nonlinear term, representing the reaction), is not satisfied. This is again primarily due to the fact that the nonlinearities may change sign. Usually this condition plays a key role in the dissipation process, and thus if it is satisfied, then it often leads to the existence of a global attractor for the system. This opposite signed nonlinearity, however, best represents the biological process at work. That is, the rate of increase of $f$ and $m$ happen with rate $g_{1}, g_{3}$, and $g_{4}$, while the loss or death of $f$ and $m$ happen with rate $g_{2}$ and $g_{5}$. From a mathematical point of view, this opposite signed coupling causes extensive problems in proving existence of a global attractor. Essentially, showing asymptotic compactness of the semigroup in question is not straightforward. Certain methods have been proposed in the literature to overcome these difficulties. Recently You in $[46,47,48]$ has studied the asymptotic dynamics of various autocatalytic reaction-diffusion systems such as Brusselator system and Gray-Scott equations. The key similarity in these systems, and the system we consider, is that the asymptotic sign condition in vector version is again not satisfied. You has devised an elegant technique, based on a Kuratowski measure decomposition, that enables one to get around this hindrance in order to prove the existence of a global attractor for the various systems he considers. Our approach in the current manuscript differs quite significantly from these decomposition methods. The Lyapunov function technique enables us to derive the $L^{p}$ bounds on the solution. Via these estimates, the $H^{1}$ bounds are derived, and subsequently the asymptotic compactness of the semigroup in question is shown, which leads to the existence of a global attractor.

The current manuscript is organized as follows. In Section 1 we have introduced the general problem of invasive species, and the relevant literature as concerns their control. In Section 2 we define certain preliminaries. In Section 3 we give the proof of global existence for the generalized system considered. In Section 4, this proof is modified to tackle a system where the reaction terms are polynomials. In Section 5, we discuss the asymptotic behavior of the system, and show the existence of a global attractor. Section 6 discusses the finite dimensionality of this attractor. In Section 7 we numerically simulate the system, to test our analytical findings. Finally we make various concluding remarks in Section 8.

\section{Notations and preliminary observations}

It is well known that to prove global existence of solutions to (1.6)-(1.8) (see Henry [14]), it suffices to derive a uniform estimate of $\left\|r_{1}(f, m, s)\right\|_{p}$ and $\left\|r_{2}(f, m, s)\right\|_{p}$ on $\left[0, T^{*}[\right.$ for some $p>N / 2$. Our aim is to construct polynomial Lyapunov functionals (see S. Kouachi and A. Youkana [24] and S. Kouachi [23]) for the solution $(f, m)$ of system (1.6)-(1.7), so as to estimate their $\mathrm{L}^{p}$-norms and thus deduce global existence.

The usual norms in spaces $\mathbb{L}^{p}(\Omega), \mathbb{L}^{\infty}(\Omega)$, and $\mathbb{C}(\bar{\Omega})$ are respectively denoted by

$$
\|u\|_{p}^{p}=\int_{\Omega}|u(x)|^{p} d x
$$




$$
\|u\|_{\infty}=\max _{x \in \Omega}|u(x)|
$$

Also, in all subsequent estimates $C, C_{i}, i=1,2, \ldots$ are generic constants that can change in value from line to line, and sometimes within the same line if so required. Since the nonlinear right hand side of (1.6)-(1.7) is continuously differentiable on $\mathbb{R}^{+} \times$ $\mathbb{R}^{+}$, then for any initial data in $\mathbb{C}(\bar{\Omega})$ or $\mathbb{L}^{p}(\Omega), p \in(1,+\infty)$, it is easy to check directly its Lipschitz continuity on bounded subsets of the domain of a fractional power of the operator

$$
\left(\begin{array}{cc}
-d_{1} \Delta & 0 \\
0 & -d_{2} \Delta
\end{array}\right)
$$

Under these assumptions, the following local existence result is well known; see A. Friedman [6], D. Henry [14], A. Pazy [37], and J. Smoller [42].

Theorem 2.1. The system (1.6)-(1.8) admits a unique, classical solution $(f, m, s)$ on $\left[0, T_{\max }\left[\times \Omega\right.\right.$. If $T_{\max }<\infty$, then

$$
\lim _{t \succ T_{\max }}\left\{\|f(t, .)\|_{\infty}+\|m(t, .)\|_{\infty}+\|s(t, .)\|_{\infty}\right\}=\infty,
$$

where $T_{\max }$ denotes the eventual blowing-up time in $\mathbb{L}^{\infty}(\Omega)$.

\section{Statement and proof of the main results}

For the global existence of the system of coupled equations (1.6)-(1.7), we introduce the following functional used in S. Kouachi [26]:

$$
t \longrightarrow L(t)=\int_{\Omega} \sum_{i=0}^{p} C_{p}^{i} \theta_{i} f^{i} m^{p-i} d x,
$$

where $\left\{\theta_{i}\right\}_{i \in \mathbb{N}}$ is a real positive increasing sequence satisfying

$$
\frac{\theta_{i} \theta_{i+2}}{\theta_{i+1}^{2}} \geq d, i=0,1, \ldots p-2
$$

where

$$
d>\frac{\left(d_{1}+d_{2}\right)^{2}}{4 d_{1} d_{2}} .
$$

Remark 3.1. Condition (3.2) implies that

$$
\theta_{i} \geq K d^{i^{2}}, i=0,1, \ldots
$$

For the reaction terms we suppose that

$$
\lim _{|f|+|m|+|s| \rightarrow+\infty} \frac{g_{2} g_{5}}{g_{1}\left(g_{3} m+g_{4} s\right) f}=+\infty,
$$

or

$$
\lim _{|f|+|m| \rightarrow+\infty} \frac{g_{3} m+g_{4} s}{g_{2}}<+\infty \text { for all } s \geq 0 \text { and } \lim _{|f|+|m|+|s| \rightarrow+\infty} \frac{g_{5}}{g_{1} f}=+\infty .
$$

The main result of the paper is the following theorem. 
THEOREM 3.1. If $(f(t,),. m(t,)$.$) is any positive solution of the problem (1.6)-$ (1.7), then under conditions (3.2)-(3.4) or (3.5), the functional $L(t)$ given by (3.1) is decreasing on the interval $\left[0, T_{\max }[\right.$.

Proof. By differentiating $L$ with respect to $t$ and then by simple use of Green's formula we obtain

$$
L^{\prime}(t)=I+J
$$

where

$$
\begin{aligned}
I=-p(p-1) \sum_{i=0}^{p-2} C_{p-2}^{i} \int_{\Omega} f^{i} m^{p-2-i}\left(d_{1} \theta_{i+2}|\nabla f|^{2}\right. \\
\left.\quad+\left(d_{1}+d_{2}\right) \theta_{i+1} \nabla f \nabla m+d_{2} \theta_{i}|\nabla m|^{2}\right) d x
\end{aligned}
$$

and

$$
J=\int_{\Omega}\left[p \sum_{i=0}^{p-1}\left(\theta_{i+1} r_{1}(f, m, s)+\theta_{i} r_{2}(f, m, s)\right) C_{p-1}^{i} f^{i} m^{p-1-i}\right] d x .
$$

By choosing a sequence satisfying (3.2) and (3.3), it is easy to verify that the quadratic form with respect to $(\nabla f, \nabla m)$ is positive, which implies $I \leq 0$.

For the second integral we have

$$
\begin{aligned}
& \theta_{i+1}\left(g_{1} f m-g_{2} f\right)+\theta_{i}\left(g_{3} f m+g_{4} f s-g_{5} m\right) \\
= & {\left[\theta_{i+1} g_{1} f m-\theta_{i} g_{5} m\right]+\left[-\theta_{i+1} g_{2} f+\theta_{i}\left(g_{3} f m+g_{4} f s\right)\right] } \\
= & {\left[\theta_{i+1} g_{1} f-\theta_{i} g_{5}\right] m+\left[-\theta_{i+1} g_{2}+\theta_{i}\left(g_{3} m+g_{4} s\right)\right] f, }
\end{aligned}
$$

so that $J \leq 0$ if we choose

$$
\theta_{i+1} g_{1} f-\theta_{i} g_{5} \leq 0 \text { and }-\theta_{i+1} g_{2}+\theta_{i}\left(g_{3} m+g_{4} s\right) \leq 0,
$$

which can be written as

$$
\frac{g_{3} m+g_{4} s}{g_{2}} \leq \frac{\theta_{i+1}}{\theta_{i}} \leq \frac{g_{5}}{g_{1} f}
$$

Under conditions (1.12) and (3.4), the interval $\left[\frac{g_{3} m+g_{4} s}{g_{2}}, \frac{g_{5}}{g_{1} f}\right]$ becomes sufficiently large. We choose the sequence $\left\{\theta_{i}\right\}$ satisfying (3.10) and (3.2) at the same time. This proves the theorem.

By application of the preliminary observations, we have the following result.

COROLlary 3.2. If the reaction terms are continuously differentiable on $\mathbb{R}_{+}^{3}$, then all positive solutions of (1.6)-(1.9) with initial data in $\mathbb{L}^{p}(\Omega)$ are in $\mathbb{L}^{\infty}\left(0, T^{*} ; \mathbb{L}^{p}(\Omega)\right)$ for all $p \geq 1$.

Proof. If $p$ is an integer, the proof is an immediate consequence of Theorem 3.1 and the trivial inequality

$$
\int_{\Omega}(d f(t, x)+m(t, x))^{p} d x \leq C_{1} L(t) \text { on }\left[0, T^{*}[, i=0,1, \ldots, p,\right.
$$


where $C_{1}$ is a positive constant depending on $p$. In the general case one uses, besides the well known Hölder inequality,

$$
\int_{\Omega}(d f(t, x)+m(t, x))^{p} d x \leq(|\Omega|)^{\frac{r-p}{r}}\left(\int_{\Omega}(d f(t, x)+m(t, x))^{r}\right)^{\frac{p}{r}}, i=0,1, \ldots, p,
$$

where $r$ is the first integer such that $r \geq p$, and $|\Omega|$ is the measure of the set $\Omega$.

If we suppose that the reaction terms are of polynomial growth,

$$
\left|g_{i}(f, m, s)\right| \leq C_{2}(f, m, s)[1+f+m+s]^{l} \text { on } \mathbb{R}_{+}^{3}, i=1, \ldots, 6,
$$

where $C_{2}$ is a positive and bounded function on bounded subsets of $\mathbb{R}_{+}^{3}$, then we have the following result.

Proposition 3.3. If the reaction terms are of polynomial growth satisfying conditions (1.11) and (3.4), then all positive solutions of (1.6)-(1.9) with initial data in $\mathbb{L}^{\infty}(\Omega)$ are global.

Proof. From Corollary 3.2, there exists a positive constant $C_{3}$ such that

$$
\int_{\Omega}(f(t, x)+m(t, x)+1)^{p} d x \leq C_{3} \text { on }\left[0, T_{\max }[\right.
$$

for all $p \geq 1$, and from (3.13) we have

$$
\left|r_{i}(f, m, s)\right|^{\frac{p}{l+2}} \leq C_{2}(f, m, s)(f+m+1)^{p} \text { on }\left[0, T_{\max }[\times \Omega, i=1, \ldots, 6 .\right.
$$

Since $f$ and $m$ are in $\mathbb{L}^{\infty}\left(0, T^{*} ; \mathbb{L}^{p}(\Omega)\right)$ for all $p \geq 1$, we can choose $p \geq 1$ such that $\frac{p}{l+2}>\frac{N}{2}$, and therefore from the preliminary observations the solution is global.

REMARK 3.2. In the case when condition (3.4) is not satisfied, we can prove global existence under more general conditions by changing (3.10) to

$$
\theta_{i+1} g_{1} f-\theta_{i} g_{5} \leq K \theta_{i} \text { and }-\theta_{i+1} g_{2}+\theta_{i}\left(g_{3} m+g_{4} s\right) \leq K \theta_{i+1},
$$

so that (3.10) becomes

$$
\frac{g_{3} m+g_{4} s}{g_{2}+K} \leq \frac{\theta_{i+1}}{\theta_{i}} \leq \frac{g_{5}+K}{g_{1} f}
$$

\section{The case of polynomial reaction terms}

If the reaction terms have polynomial growth

$$
\left\{\begin{array}{l}
r_{1}(f, m, s)=a_{1} f^{\alpha_{1}+1} m^{\beta_{1}+1}-a_{2} f^{\alpha_{2}+1} m^{\beta_{2}} \\
r_{2}(f, m, s)=a_{3} f^{\alpha_{3}+1} m^{\beta_{3}+1}+a_{4} f^{\alpha_{4}+1} m^{\beta_{4}} s-a_{5} f^{\alpha_{5}} m^{\beta_{5}+1} \\
r_{3}(f, m, s)=a_{6} s^{q}-a_{7} s^{p}
\end{array}\right.
$$

that is

$$
\begin{aligned}
& g_{1}=a_{1} f^{\alpha_{1}} m^{\beta_{1}}, g_{2}=a_{2} f^{\alpha_{2}+1} m^{\beta_{2}}, g_{3}=a_{3} f^{\alpha_{3}} m^{\beta_{3}} \\
& g_{4}=a_{4} f^{\alpha_{4}} m^{\beta_{4}} s, g_{5}=a_{5} f^{\alpha_{5}} m^{\beta_{5}+1}
\end{aligned}
$$


where $\alpha_{i}, \beta_{i}$, and $a_{i}$ are positive reals. Condition (3.4) can be written as follows:

$$
\lim _{|f|+|m| \rightarrow+\infty} \frac{\left(a_{3} f^{\alpha_{3}} m^{\beta_{3}+1}+a_{4} f^{\alpha_{4}} m^{\beta_{4}} s\right)}{a_{2} f^{\alpha_{2}} m^{\beta_{2}}}<+\infty \text { and } \lim _{|f|+|m| \rightarrow+\infty} \frac{a_{5} f^{\alpha_{5}} m^{\beta_{5}}}{a_{1} f^{\alpha_{1}+1} m^{\beta_{1}}}=+\infty,
$$

which are satisfied if we choose

$$
\alpha_{2} \geq \max \left\{\alpha_{3}, \alpha_{4}\right\}, \beta_{2} \geq \max \left\{1+\beta_{3}, \beta_{4}\right\}, \alpha_{5} \geq 1+\alpha_{1}, \text { and } \beta_{5} \geq \beta_{1} .
$$

For equation (1.8) with homogeneous Neumann boundary conditions, standard methods give global existence if we choose

$$
q \leq 1 \text { for all } p \geq 0 \text { or } p>q>1 .
$$

The first condition implies, by comparison methods, that

$$
s(t, .) \leq \bar{s}(t, .),
$$

where $\bar{s}(t,$.$) is the solution of the following problem:$

$$
\left\{\begin{aligned}
\partial_{t} \bar{s}-d_{3} \Delta \bar{s} & =a_{6} \bar{s}^{q}, & & \text { on } \mathbb{R}^{+} \times \Omega, \\
\partial_{\eta} \bar{s} & =0, & & \text { on } \mathbb{R}^{+} \times \partial \Omega, \\
\bar{s}(0, .) & =\max _{x \in \Omega}(0, x), & & \text { on } \Omega
\end{aligned}\right.
$$

which exists globally in time and is bounded on $[[0, T][\times \Omega$ for all $T>0$.

For the second condition, we apply Young's inequality to the term $s^{q}$ to get

$$
s^{q}=s^{q_{1}+q_{2}} \leq \frac{a_{7}}{a_{6}} s^{\gamma_{1} q_{1}}+C_{6} s^{\gamma_{2} q_{2}},
$$

where

$$
\frac{1}{\gamma_{1}}+\frac{1}{\gamma_{2}}=1 \text { with } \gamma_{1}, \gamma_{2}>1, \gamma_{1} q_{1}=p, \text { and } \gamma_{2} q_{2}=1,
$$

and this is satisfied if we choose

$$
\gamma_{1}=\frac{p-1}{q-1} \text { and } \gamma_{2}=\frac{p-1}{p-q}
$$

(4.8) implies that

$$
r_{3} \leq C_{7} s, \text { for all } s \geq 0,
$$

which gives global existence and boundedness of $s(t,$.$) .$

Now we can state the following proposition.

Proposition 4.1. If the constants $\alpha_{i}, \beta_{i}, i=1, \ldots, 5$, and $p, q$, are positive reals which satisfy conditions (4.4), (4.5), then for all positive parameters $a_{j}, j=1, \ldots, 7$, and all positive initial data in $\mathbb{L}^{\infty}(\Omega)$, the solutions of (1.6)-(1.9) with reaction terms given by (4.1) are global.

Proof. The proof is an immediate consequence of Proposition 3.1 and conditions (4.4) and (4.5). 


\section{Asymptotic behavior and global attractor}

In this section we will prove the existence of a global attractor for the reaction diffusion system (1.6)-(1.9). Recall the following definition.

Definition 5.1. Consider a semigroup $S(t)$ acting on a reflexive Banach space $H$. Then the global attractor $\mathcal{A} \subset H$ for this semigroup is an object that has the following properties:

i) $\mathcal{A}$ is compact in $H$;

ii) $\mathcal{A}$ is invariant, i.e. $S(t) \mathcal{A}=\mathcal{A}, t \geq 0$;

iii) If $B$ is bounded in $H$ then

$$
\operatorname{dist}_{H}(S(t) B, \mathcal{A}) \rightarrow 0, t \rightarrow \infty .
$$

Next various preliminaries are presented, detailing the phase spaces of interest and recalling certain standard theory. Let us define our phase spaces of interest.

$$
H=L^{2}(\Omega) \times L^{2}(\Omega) \times L^{2}(\Omega), V=H^{1}(\Omega) \times H^{1}(\Omega) \times H^{1}(\Omega) .
$$

In order to prove the existence of a global attractor we are required to show:

i) There exists a bounded absorbing set in the phase space.

ii) The asymptotic compactness property of the semigroup in question, [44, 43].

These notions are defined next.

Definition 5.2 (Bounded absorbing set). $\quad A$ bounded set $\mathcal{B}$ in a reflexive Banach space $H$ is called a bounded absorbing set if for each bounded subset $U$ of $H$, there is a time $T=T(U)$, such that $S(t) U \subset \mathcal{B}$ for all $t>T$. The number $T=T(U)$ is referred to as the compactification time for $S(t) U$. This is essentially the time after which the semigroup compactifies.

Definition 5.3 (Asymptotic compactness). The semigroup $\{S(t)\}_{t \geq 0}: H \rightarrow H$ associated with a dynamical system is said to be asymptotically compact in $H$ if for any $\left\{u_{i 0, n}\right\}_{n=1}^{\infty}$ bounded in $H$, and a sequence of times $\left\{t_{n} \rightarrow \infty\right\}, S\left(t_{n}\right) u_{i 0, n}$ possesses a convergent subsequence in $H$.

We will use the functional $L$ to show the compactness of the trajectories $\{f(t, .), m(t, .), s(t, .)\}_{t \geq 0}$ in the spaces $\mathbb{L}^{p}(\Omega)$. Using the conditions (3.3) and (3.4), we can find a constant $C_{4}$ such that

$$
I \geq p(p-1) C_{4} \int_{\Omega}\left((d f+m)^{p-2}|\nabla(d f+m)|^{2}\right) d x
$$

and this gives

$$
L^{\prime}(t)+p(p-1) C_{4} \int_{\Omega}\left((d f+m)^{p-2}|\nabla(d f+m)|^{2}\right) d x \leq 0
$$

by integration with respect to $t$. We then deduce using (3.11) that

$$
\int_{\Omega}(d f(t, x)+m(t, x))^{p} d x+C_{5} \int_{0}^{t} \int_{\Omega}\left((d f+m)^{p-2}|\nabla(d f+m)|^{2}\right) d x d \sigma \leq L(0) .
$$


Since $d$ arbitrarily satisfies condition (3.4), the above inequality yields

$$
f(t, .), m(t, .) \in \mathbb{L}^{\infty}\left(\left[0, \infty\left[; \mathbb{L}^{p}(\Omega)\right) \cap \mathbb{L}^{2}\left(\left[0, \infty\left[; \mathbb{H}^{1}(\Omega)\right) .\right.\right.\right.\right.
$$

Now, specifically, we will first show a bounded absorbing set in the phase space $L^{2}(\Omega)$. We see that (5.3), with $p=2$, yields

$$
\|f\|_{2}^{2} \leq C_{1}, \forall t \geq t_{1} .
$$

Note that here $C_{1}$ is independent of time and the initial data, and $t_{1}$ is the compactification time that depends on the initial data, easily determined via Gronwall's inequality. We next aim to show the asymptotic compactness. Let us begin by multiplying (1.6) by $f$, and integrating by parts over $\Omega$ to obtain

$$
\frac{1}{2} \frac{d}{d t}\|f\|_{2}^{2}+\|\nabla f\|_{2}^{2}=a_{1} \int_{\Omega} f^{\alpha_{1}+2} m^{\beta_{1}+1} d x-a_{2} \int_{\Omega} f^{\alpha_{2}+2} m^{\beta_{2}} d x .
$$

We now use the positivity of $f, m$ along with Hölder's inequality to obtain

$$
\frac{1}{2} \frac{d}{d t}\|f\|_{2}^{2}+\|\nabla f\|_{2}^{2} \leq a_{1}\|f\|_{2\left(\alpha_{1}+2\right)}^{\alpha_{1}+2}\|m\|_{2\left(\beta_{1}+1\right)}^{\beta_{1}+1} \leq C .
$$

This follows via the a priori $L^{p}$ bound on the solutions, from (5.3), and hence in particular for $p=\max \left(2\left(\alpha_{1}+2\right), 2\left(\beta_{1}+1\right)\right)$. Note that the $L^{p}$ bounds follow for $L^{2}$ initial data by the regularizing property of the semigroup [37]. Now we integrate (5.7) in the time interval from $[t, t+1]$ for any $t \geq t_{1}$ to obtain

$$
\|f(t+1)\|_{2}^{2}+\int_{t}^{t+1}\|\nabla f\|_{2}^{2} d s \leq C_{5}+\|f(t)\|_{2}^{2} \leq C_{6}, \forall t \geq t_{1} .
$$

Thus we have the following uniform integral in time bound:

$$
\int_{t}^{t+1}\|\nabla f\|_{2}^{2} d s \leq C_{6}, \forall t \geq t_{1}
$$

and using the mean value theorem for integrals we obtain the bound

$$
\left\|\nabla f\left(t^{*}\right)\right\|_{2}^{2} d s \leq C_{6}, \forall t, t^{*} \in[t, t+1], \forall t \geq t_{1} .
$$

We next multiply (1.6) by $-\Delta f$ and integrate by parts over $\Omega$ to obtain

$$
\frac{1}{2} \frac{d}{d t}\|\nabla f\|_{2}^{2}+\|\Delta f\|_{2}^{2}=a_{1} \int_{\Omega} f^{\alpha_{1}+1} m^{\beta_{1}+1}(-\Delta f) d x-a_{2} \int_{\Omega} f^{\alpha_{2}+1} m^{\beta_{2}}(-\Delta f) d x .
$$

Then employing Young's inequality yields

$$
\begin{aligned}
\frac{1}{2} \frac{d}{d t}\|\nabla f\|_{2}^{2}+\|\Delta f\|_{2}^{2} \leq \frac{1}{4} \| \Delta & f\left\|_{2}^{2}+2\right\| f\left\|_{4\left(\alpha_{1}+1\right)}^{4\left(\alpha_{1}+1\right)}+2\right\| m \|_{4\left(\beta_{1}+1\right)}^{4\left(\beta_{1}+1\right)} \\
& +\frac{1}{4}\|\Delta f\|_{2}^{2}+2\|f\|_{4\left(\alpha_{2}+1\right)}^{4\left(\alpha_{2}+1\right)}+2\|m\|_{4\left(\beta_{2}\right)}^{4\left(\beta_{2}\right)}
\end{aligned}
$$

which, via the a priori $L^{p}$ bounds on the solutions, and hence in particular for $p=\max \left(4\left(\alpha_{1}+1\right), 4\left(\beta_{1}+1\right), 4\left(\alpha_{2}+1\right), 4\left(\beta_{2}\right)\right)$, leads to

$$
\frac{d}{d t}\|\nabla f\|_{2}^{2}+\|\Delta f\|_{2}^{2} \leq 4\|f\|_{4\left(\alpha_{1}+1\right)}^{4\left(\alpha_{1}+1\right)}+4\|m\|_{4\left(\beta_{1}+1\right)}^{4\left(\beta_{1}+1\right)}+4\|f\|_{4\left(\alpha_{2}+1\right)}^{4\left(\alpha_{2}+1\right)}+4\|m\|_{4\left(\beta_{2}\right)}^{4\left(\beta_{2}\right)} \leq C .
$$


Now using the Sobolev embedding of $H^{2}(\Omega) \hookrightarrow H^{1}(\Omega)$ we obtain

$$
\frac{d}{d t}\|\nabla f\|_{2}^{2}-C_{1}\|\nabla f\|_{2}^{2} \leq C .
$$

The uniform Gronwall lemma [44] via integration in the time interval $\left[t^{*}, t\right]$ yields the following uniform bound:

$$
\|\nabla f\|_{2}^{2} \leq \frac{C}{C_{1}}+C_{6}, \forall t \geq t^{*}
$$

This follows via (5.9), (5.10).

We now state the following theorem.

THEOREM 5.4. Consider the reaction diffusion system described via (1.6)-(1.9). Under conditions (3.2)-(3.4) or (3.5), there exists a $(H, H)$ global attractor $\mathcal{A}$ for the system. This is compact and invariant in $H$, and it attracts all bounded subsets of $H$ in the $H$ metric.

Proof. We have shown global existence via Proposition 3.3. Thus there exists a well defined semigroup $\{S(t)\}_{t \geq 0}: H \rightarrow H$. The equation (5.5) demonstrates the existence of bounded absorbing sets in $H$. Thus given a sequence $\left\{f_{0, n}\right\}_{n=1}^{\infty}$ that is bounded in $L^{2}(\Omega)$, we know that for $t>t^{*}$ (where $t^{*}$ appears in (5.10)),

$$
S(t)\left(f_{0, n}\right) \subset B \subset H^{1}(\Omega) .
$$

Here $B$ is the bounded absorbing set in $H^{1}(\Omega)$. Now for n large enough $t_{n}>t^{*}$, and thus for such $t_{n}$ we have

$$
S\left(t_{n}\right)\left(f_{0, n}\right) \subset B \subset H^{1}(\Omega) .
$$

This implies that we have the following uniform bound, via (5.14):

$$
\left|S\left(t_{n}\right)\left(f_{0, n}\right)\right|_{H^{1}(\Omega)} \leq C,
$$

which implies via standard functional analysis theory (see [44, 43]) the existence of a subsequence still labelled $S\left(t_{n}\right)\left(f u_{0, n}\right)$ such that

$$
S\left(t_{n}\right)\left(f_{0, n}\right) \rightarrow f \text { in } H^{1}(\Omega) .
$$

This yields, via the compact Sobolev embedding of

that

$$
V \hookrightarrow H,
$$

$$
S\left(t_{n}\right)\left(f_{0, n}\right) \rightarrow f \text { in } L^{2}(\Omega) .
$$

Analogous estimates and convergences can be derived for the $m$ and $s$ components. This yields the asymptotic compactness of the semigroup $\{S(t)\}_{t>0}$ in $H$. The theorem is now proved.

REMARK 5.1. It is interesting to note that the attractor is a one point attractor, that is given a set of parameters, the solution converges in long time to a steady state. This may not be a spatially uniform state. However, it supports the extinction state for an appropriate range of parameters, thus validating the success of the control strategy. The details of this can be seen in Section 7 . 


\section{Finite Dimensionality of the global attractor}

In this section we show that the Hausdorff and fractal dimensions of the global attractor for the reaction diffusion system (1.6)-(1.9) are finite. Recall the following definitions.

Definition 6.1 (Fractal dimension). Consider a subset $X$ of a Banach space $H$. If $\bar{X}$ is compact, the fractal dimension of $X$, denoted $d_{f}(X)$, is given by

$$
d_{f}(X)=\limsup _{\epsilon \rightarrow 0} \frac{\log N(X, \epsilon)}{\log \left(\frac{1}{\epsilon}\right)} .
$$

Here $N(X, \epsilon)$ denotes the minimum number of closed balls of radius $\epsilon$, required to cover $X$. Note $d_{f}(X)$ can take the value $+\infty$.

Definition 6.2 (Hausdorff dimension). Consider a subset $X$ of a Banach space $H$. If $\bar{X}$ is compact, the Hausdorff dimension of $X$, denoted $d_{H}(X)$, is given by

$$
d_{H}(X)=\inf _{d>0}\left\{d: \mathcal{H}^{d}(X)=0\right\} .
$$

Here

$$
\mathcal{H}^{d}(X)=\lim _{\epsilon \rightarrow 0} \mu(X, d, \epsilon)
$$

where

$$
\mu(X, d, \epsilon)=\inf \left\{\sum_{i} r_{i}^{d}: r_{i} \leq \epsilon \text { and } X \subseteq \cup_{i} B\left(x_{i}, r_{i}\right)\right\}
$$

and $B\left(x_{i}, r_{i}\right)$ are balls with radius $r_{i}$.

We will provide upper bounds on these dimensions in terms of parameters in the model. There is a standard methodology to derive these estimates. We consider a volume element in the phase space, and try and derive conditions that will cause it to decay as time goes forward. If $\mathcal{A}$ is the global attractor of the semigroup $\{S(t)\}_{t \geq 0}$ in $H$ associated with (1.6)-(1.9), we can define

$$
q_{n}(t)=\sup _{u_{0} \in A} \sup _{g_{i} \in H,\left\|g_{i}\right\|=1,1 \leq i \leq n} \frac{1}{t} \int_{0}^{t} \operatorname{Tr}\left(\Delta U(\tau)+F^{\prime}\left(S(\tau) u_{0}\right) \circ Q_{n}(\tau) d \tau,\right.
$$

where

$$
q_{n}=\limsup _{t \rightarrow \infty} q_{n}(t) .
$$

Here $\mathrm{F}$ is the nonlinear map in (1.6)-(1.9). $Q_{n}$ is the orthogonal projection of the phase space $H$ onto the subspace spanned by $U_{1}(t), U_{2}(t), \cdots, U_{n}(t)$, with

$$
U_{i}(t)=L\left(S(t) u_{0}\right) g_{i}, i=1,2, \ldots, n .
$$

$L\left(S(t) u_{0}\right)$ is the Fréchet derivative of the map $S(t)$ at $u_{0}$, with $\mathrm{t}$ fixed. Also for this model, $L\left(S(t) u_{0}\right) g=U(t)=(F, M, S)$, where $u=(f, m, s)$ is a solution to (1.6)-(1.9), $\phi_{j}=\left(\phi_{j}^{1} \ldots \phi_{j}^{3},\right)$ are an orthonormal basis for the subspace $Q_{n}(\tau) H$, and $(F, M, S)$ are strong solutions to the variational equations for the reaction diffusion system (1.6)(1.9). These are given by

$$
\frac{\partial F}{\partial t}=\Delta F+a_{1}\left(\beta_{1}+1\right) f^{\alpha_{1}+1} m^{\beta_{1}} M+a_{1}\left(\alpha_{1}+1\right) f^{\alpha_{1}} m^{\beta_{1}+1} F
$$




$$
\begin{gathered}
-a_{2}\left(\beta_{2}\right) f^{\alpha_{2}+1} m^{\beta_{2}-1} M-a_{2}\left(\alpha_{2}+1\right) f^{\alpha_{2}} m^{\beta_{2}} F \\
\frac{\partial M}{\partial t}=\Delta M+a_{3}\left(\beta_{3}+1\right) f^{\alpha_{3}+1} m^{\beta_{3}} M+a_{3}\left(\alpha_{3}+1\right) f^{\alpha_{3}} m^{\beta_{3}+1} F \\
+a_{4} f^{\alpha_{4}+1}\left[m^{\beta_{4}} S+\beta_{4} s m^{\beta_{4}-1} M\right]+a_{4}\left(\alpha_{4}+1\right) m^{\beta_{4}} s f^{\alpha_{4}} F \\
-a_{5}\left(\beta_{5}+1\right) f^{\alpha_{5}} m^{\beta_{5}+1} M-a_{5}\left(\alpha_{5}\right) f^{\alpha_{5}-1} m^{\beta_{5}+1} F \\
\frac{\partial S}{\partial t}=\Delta S+a_{6} q s^{q-1} S-a_{7} p s^{p-1} S, \\
F(0)=\eta, M(0)=\xi, S(0)=\zeta .
\end{gathered}
$$

We recall the following lemma from [44], which will be useful to derive the requisite estimates.

LEMMA 6.3. If there is an integer $n$ such that $q_{n}<0$, then the Hausdorff and fractal dimensions of $\mathcal{A}$, denoted $d_{H}(\mathcal{A})$ and $d_{F}(\mathcal{A})$, satisfy the following estimates:

$$
\begin{aligned}
& d_{H}(\mathcal{A}) \leq n, \\
& d_{F}(\mathcal{A}) \leq 2 n .
\end{aligned}
$$

Our aim is thus clear. We will derive exactly the conditions that enforce $q_{n}<0$ for (6.5). However, without loss of generality, we will assume $d_{1}=1, d_{2}=1, d_{3}=1$. We begin our estimates.

$$
\begin{aligned}
& \operatorname{Tr}\left(\Delta U(\tau)+F^{\prime}\left(S(\tau) u_{0}\right) \circ Q_{n}(\tau)\right. \\
= & \sum_{j=1}^{n} 3\left\langle\Delta \phi_{j}(\tau), \phi_{j}(\tau)\right\rangle+\left\langle F^{\prime}\left(S(\tau) u_{0}\right) \phi_{j}(\tau), \phi_{j}(\tau)\right\rangle \\
= & -3 \sum_{j=1}^{n}\left|\nabla \phi_{j}(\tau)\right|^{2}+J_{1}+J_{2}+J_{3} .
\end{aligned}
$$

Here

$$
\begin{aligned}
J_{1} \leq & \sum_{j=1}^{n} \int_{\Omega} a_{1}\left(\beta_{1}+1\right) f^{\alpha_{1}+1} m^{\beta_{1}} \phi_{j}^{1}(\tau) \phi_{j}^{2}(\tau)+a_{1}\left(\alpha_{1}+1\right) f^{\alpha_{1}} m^{\beta_{1}+1}\left|\phi_{j}^{1}(\tau)\right|^{2} \\
& \quad-a_{2}\left(\beta_{2}\right) f^{\alpha_{2}+1} m^{\beta_{2}-1} \phi_{j}^{1}(\tau) \phi_{j}^{2}(\tau)-a_{2}\left(\alpha_{2}+1\right) f^{\alpha_{2}} m^{\beta_{2}}\left|\phi_{j}^{1}(\tau)\right|^{2} \\
\leq & C|f|_{4 \alpha_{1}}^{\alpha_{1}}|m|_{4\left(\beta_{1}+1\right)}^{\beta_{1}+1}\left|\phi_{j}^{1}(\tau)\right|_{4}^{2}+C_{1}|f|_{8\left(\alpha_{2}+1\right)}^{4 \alpha_{1}}|m|_{8 \beta_{2}}^{4 \beta_{2}}\left|\phi_{j}^{1}(\tau)\right|_{4}\left|\phi_{j}^{2}(\tau)\right|_{2} \\
\leq & C \sum_{j=1}^{n}\left|\phi_{j}(\tau)\right|_{4}^{2}+\leq C_{1} \sum_{j=1}^{n}\left|\phi_{j}(\tau)\right|_{2}^{2} \\
\leq & C \sum_{j=1}^{n}\left|\phi_{j}(\tau)\right|_{4}^{2}+C_{1} n .
\end{aligned}
$$

Then $J_{2}$ and $J_{3}$ are estimated likewise and we obtain

$$
J_{2} \leq C \sum_{j=1}^{n}\left|\phi_{j}(\tau)\right|_{4}^{2}+C_{2} n
$$


and

$$
J_{3} \leq C \sum_{j=1}^{n}\left|\phi_{j}(\tau)\right|_{4}^{2}+C_{3} n .
$$

Recall the Gagliardo-Nirenberg interpolation inequality [43],

$$
|\phi|_{W^{k, p}(\Omega)} \leq C|\phi|_{W^{k, p}(\Omega)}^{\theta}|\phi|_{L^{r}(\Omega)}^{1-\theta}, \text { for } \phi \in W^{m, q}(\Omega),
$$

provided $p, q, r \geq 1,0<\theta<1$, and

$$
k-\frac{n}{p} \leq \theta\left(m-\frac{n}{q}\right)-(1-\theta) \frac{n}{r}, \text { where } n=\Omega .
$$

Now we consider exponents such that,

and

$$
W^{k, p}(\Omega)=L^{4}(\Omega), W^{m, q}(\Omega)=H^{1}(\Omega), L^{r}(\Omega)=L^{2}(\Omega),
$$

$$
\theta=\frac{3}{4}
$$

Thus we obtain

$$
\left|\phi_{j}(\tau)\right|_{4}^{2} \leq\left|\nabla \phi_{j}(\tau)\right|_{2}^{\frac{3}{4}}\left|\phi_{j}(\tau)\right|_{2}^{\frac{1}{4}} \leq C\left|\nabla \phi_{j}(\tau)\right|_{2}^{\frac{3}{4}} .
$$

Using Young's inequality on the above, we obtain

$$
C\left|\nabla \phi_{j}(\tau)\right|_{2}^{\frac{3}{4}} \leq 2\left|\nabla \phi_{j}(\tau)\right|_{2}^{2}+C n,
$$

where $C$ depends on the various parameters above. Thus we obtain the estimate

$$
\begin{aligned}
& \operatorname{Tr}\left(\Delta U(\tau)+F^{\prime}\left(S(\tau) u_{0}\right) \circ Q_{n}(\tau)\right. \\
\leq & -3 \sum_{j=1}^{n}\left|\nabla \phi_{j}(\tau)\right|_{2}^{2}+2\left|\nabla \phi_{j}(\tau)\right|_{2}^{2}+C n .
\end{aligned}
$$

Now, via the generalized Sobolev-Lieb-Thirring inequalities [44], we obtain

$$
\sum_{j=1}^{n}\left|\nabla \phi_{j}(\tau)\right|_{2}^{2} \geq K_{1} \frac{n^{\frac{5}{3}}}{|\Omega|^{\frac{2}{3}}} .
$$

Here $K_{1}$ depends only on the shape and dimension of $\Omega$. Thus we obtain

$$
\begin{aligned}
& \operatorname{Tr}\left(\Delta U(\tau)+F^{\prime}\left(S(\tau) u_{0}\right) \circ Q_{n}(\tau)\right. \\
\leq & -K_{1} \frac{n^{\frac{5}{3}}}{|\Omega|^{\frac{2}{3}}}+C n
\end{aligned}
$$

for $\tau>0, u_{0} \in \mathcal{A}$, where $C=C\left(a_{i}, b_{i}, \alpha_{i}, \beta_{i}\right), i=1,2, \ldots$.

We now obtain

$$
q_{n}(t)=\sup _{u_{0} \in A g_{i} \in H,\left\|g_{i}\right\|=1,1 \leq i \leq n} \frac{1}{t} \int_{0}^{t} \operatorname{Tr}\left(\Delta U(\tau)+F^{\prime}\left(S(\tau) u_{0}\right) \circ Q_{n}(\tau) d \tau\right.
$$




$$
\leq-K_{1} \frac{n^{\frac{5}{3}}}{|\Omega|^{\frac{2}{3}}}+C\left(a_{i}, b_{i}, \alpha_{i}, \beta_{i}\right) n \forall t>0
$$

This yields

$$
q_{n}=\limsup _{t \rightarrow \infty} \leq-K_{1} \frac{n^{\frac{5}{3}}}{|\Omega|^{\frac{2}{3}}}+\left(C\left(a_{i}, b_{i}, \alpha_{i}, \beta_{i}\right)\right) n<0
$$

if the integer $n$ satisfies

$$
n-1<\left(\frac{\left(C\left(a_{i}, b_{i}, \alpha_{i}, \beta_{i}\right)\right.}{K_{1}}\right)^{\frac{3}{2}}|\Omega|<n .
$$

We can now state the following result as a direct application of Lemma 6.3.

TheOREM 6.4. Consider the reaction diffusion equation described via (1.6)-(1.9). Under conditions (3.2)-(3.4) or (3.5), the global attractor $\mathcal{A}$ of the system is of finite dimension. Furthermore, explicit upper bounds for its Hausdorff and fractal dimensions are given as follows:

$$
\begin{gathered}
d_{H}(A) \leq\left(\frac{C\left(a_{i}, b_{i}, \alpha_{i}, \beta_{i}\right)}{K_{1}}\right)^{\frac{3}{2}}|\Omega|+1, \\
d_{F}(A) \leq 2\left(\frac{\left(C\left(a_{i}, b_{i}, \alpha_{i}, \beta_{i}\right)\right.}{K_{1}}\right)^{\frac{3}{2}}|\Omega|+2 .
\end{gathered}
$$

Proof. The theorem is an immediate consequence of the estimate derived in (6.24) and Lemma 6.3.

\section{Numerical simulations}

We now provide the results of numerical simulations on (1.6) -(1.9). The system is simulated via a finite difference scheme. We integrate the system of reaction-diffusion equations by the method of lines by first discretizing the second derivatives using second-order centered finite differences and then integrating the resultant system of ODEs by the explicit third order TVD Runge-Kutta method of Gottlieb and Shu [7]. For an equation written as $\mathbf{u}_{t}=\mathbf{L}(\mathbf{u})$, the Runge-Kutta method takes the form

$$
\begin{aligned}
\mathbf{u}^{1} & =\mathbf{u}^{n}+\Delta t \mathbf{L}\left(\mathbf{u}^{n}\right) \\
\mathbf{u}^{2} & =\mathbf{u}^{1}+\frac{\Delta t}{4}\left[-3 \mathbf{L}\left(\mathbf{u}^{n}\right)+\mathbf{L}\left(\mathbf{u}^{1}\right)\right] \\
\mathbf{u}^{n+1} & =\mathbf{u}^{2}+\frac{\Delta t}{12}\left[-\mathbf{L}\left(\mathbf{u}^{n}\right)-\mathbf{L}\left(\mathbf{u}^{1}\right)+8 \mathbf{L}\left(\mathbf{u}^{2}\right)\right] .
\end{aligned}
$$

The above scheme was implemented in MATLAB R2010. We simulate with various initial conditions, and try various parameters, to obtain the spatio-temporal profiles of the solutions. The reaction terms in the simulations are as in (4.1). The results are presented in figures 7.1, 7.2, 7.3. We ensure that the conditions (4.4) for global existence are met. The details of parameters used are tabulated in table 7.1. We would like to point out that all of the simulations performed have been refined several times on grids with $300,600,900$, and 1200 points. These refinements lead to the same general shape and structure of the figures. The time step was chosen so as to maintain the stability condition $d t=\lambda h^{2} / \max \left(d_{1}, d_{2}, d_{3}\right)$, where $h=\pi / m, m$ being the 

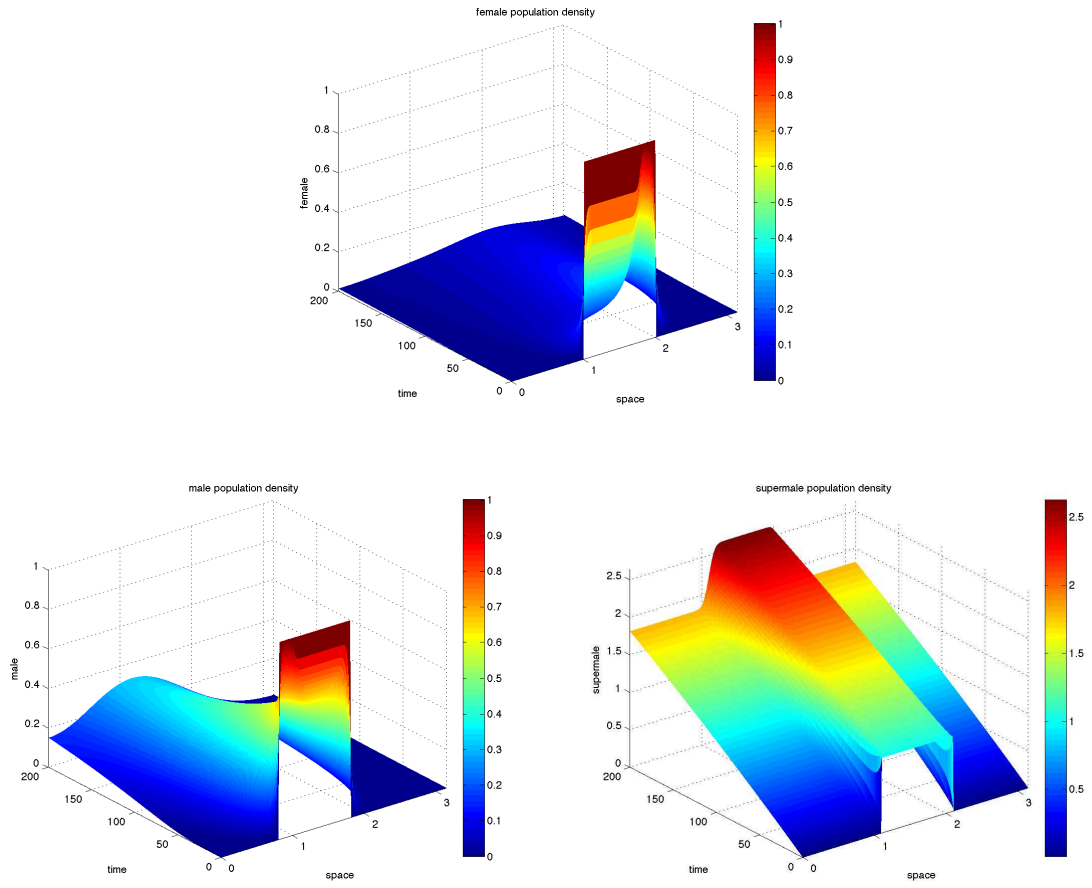

FIG. 7.1. The densities of the three species as a surface plot is shown. Uniform initial data is prescribed. The supermale is introduced as a constant source with low death rate.
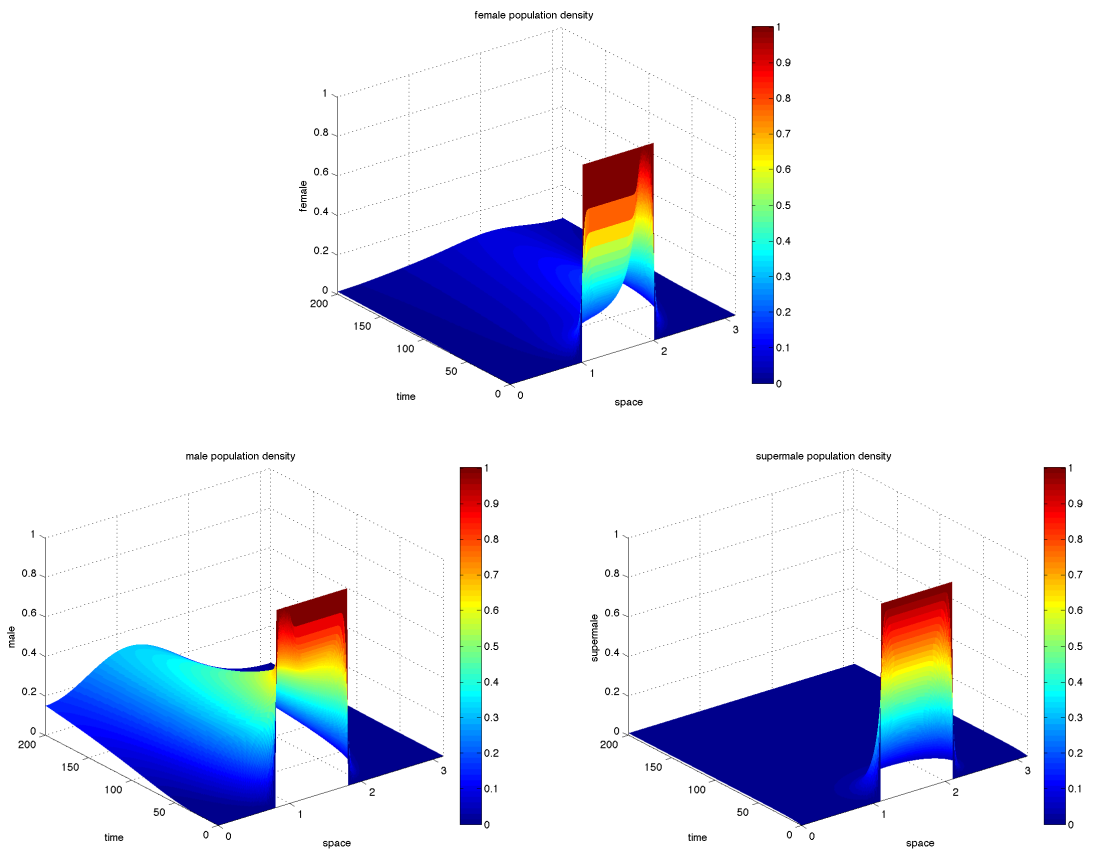

FIG. 7.2. The densities of the three species as a surface plot is shown. Uniform initial data is prescribed. The supermale is introduced as a constant source with high death rate. 

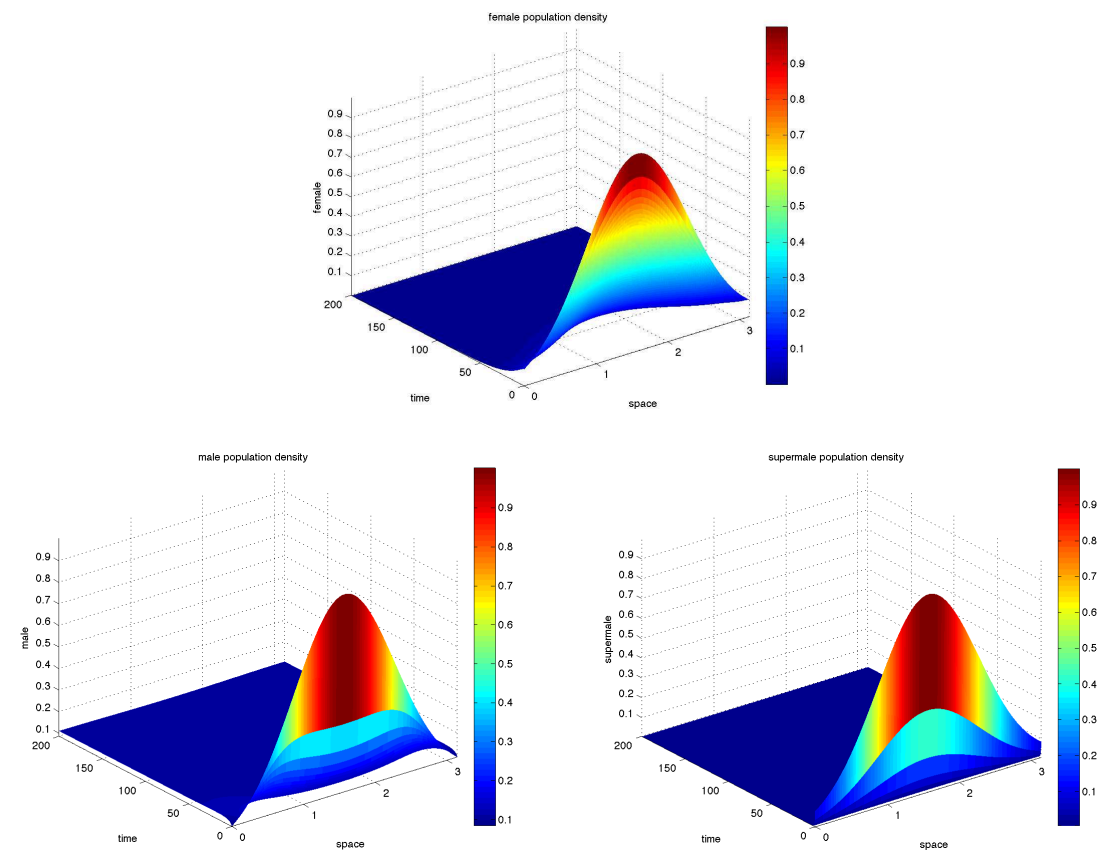

FIG. 7.3. The densities of the three species as a surface plot is shown. Uniform initial data is prescribed. Note, here the extinction state is seen.

\begin{tabular}{|c|c|c|c|}
\hline & fig 2 & fig 3 & fig 4 \\
\hline$d_{1}$ & 0.001 & 0.001 & 0.0001 \\
\hline$d_{2}$ & 0.001 & 0.001 & 0.001 \\
\hline$d_{3}$ & 0.00001 & 0.001 & 0.0001 \\
\hline$a_{1}$ & 0.01 & 0.01 & 1.5 \\
\hline$a_{2}$ & 0.8 & 0.8 & 2 \\
\hline$a_{3}$ & 0.01 & 0.01 & 0.5 \\
\hline$a_{4}$ & 0.001 & 0.001 & 0.2 \\
\hline$a_{5}$ & 0.4 & 0.4 & 5 \\
\hline$a_{6}$ & 0.01 & 0.001 & 0.01 \\
\hline$a_{7}$ & 0.001 & 0.1 & 2 \\
\hline$\alpha_{1}$ & 1 & 1 & 0 \\
\hline$\alpha_{2}$ & 1 & 1 & 0 \\
\hline$\alpha_{3}$ & 1 & 1 & 0 \\
\hline$\alpha_{4}$ & 1 & 1 & 0 \\
\hline$\alpha_{5}$ & 2 & 2 & 1 \\
\hline$\beta_{1}$ & 1 & 1 & 0 \\
\hline$\beta_{2}$ & 2 & 2 & 1 \\
\hline$\beta_{3}$ & 1 & 1 & 0 \\
\hline$\beta_{4}$ & 1 & 1 & 0 \\
\hline$\beta_{5}$ & 1 & 1 & 1 \\
\hline
\end{tabular}

TABle 7.1. Parameters used in simulations. 
number of grid points (thus in our simulations we try $m=300,600,900,1200$ ). $\lambda$ needs to be small for numerical stability. In this case $\lambda<0.5$ will work. We choose $\lambda=0.4$ for a grid with 300 points. Thus the time step varied as we refined the grid several times. However, the step size for the plots shown in figures 7.1, 7.2, 7.3 is 0.0442. These plots are shown for a spatial grid of 300 points.

We notice that the asymptotic behavior is essentially a one point attractor, that is, for a given set of parameters, the solutions in long time tend towards a steady state (not necessarily spatially uniform in all components). In particular, the extinction state is also seen, via figure 7.3, thus validating the control strategy.

The results of the simulations are illustrated next.

\section{Conclusions}

The use of Trojan sex chromosomes is an approach for eradicating invasive species that have a XY sex determination system and for which it is feasible to force sex reversal. It was clearly established that extinction is possible in the supermale dynamical system as a function of the rate $\mu$ of introduction of supermales $(s)$. The TYC system depends upon parameters that can be deduced from observations, including the carrying capacity $(K)$, the death coefficient $\left(g_{1}, g_{2}, g_{3}\right)$, and the birth coefficient $\left(g_{2}\right.$, $\left.g_{5}, g_{6}\right)$. Further refinement of these parameters should be made as field data becomes available.

The existence of a bounded absorbing set indicates that for either eradication or invasion the final state of the population is stable. Via (5.14) we showed that given the initial population and values for the parameters, it is possible to find an explicit time such that for times greater than this, there exists a state of local extinction in which the population is confined to finite sized sets in $H$, i.e. there is a compact subset of the phase plane that attracts all trajectories in the dynamical system with the bounds proposed. Knowing the analytical form of these sets helps guide the exploration of the parameter space.

The analysis of global attractors can be helpful to estimate times to extinction in complex spatial domains. We have determined that for Neumann boundary conditions on a connected domain, there exists an extinction state as a result of the introduction of $s$. This is also true for Dirichlet boundary conditions. However, more complicated geometries or mixed boundary conditions could have an influence in coexistence or extinction. In order to increase the level of sophistication of the eradication strategy, the distribution of $s$ individuals could be variable in space as opposed to the constant level that has been studied, i.e. $s$ could be a population density dependent function intended to minimize the introduction of $s$ individuals and therefore minimize costs of implementation.

The viability of YY individuals remains an open question. The supermale model assumes that phenotypes are stable after maturation, but this could be problematic for species whose sex determination involves many genes, or when there is environmental pressure to feminization or masculinization. Another potential problem is hybridization with compatible species, which would extend the eradication pressure beyond the initial target; however, this effect should disappear by the interruption of the influx of $s$.

All in all, we have shown that introduction of phenotypically manipulated supermales into a established population can lead to local extinction. Moreover, this can be done even if the population dynamics of the species involved is not governed by a logistic type control term. This theoretical result is an assurance for biologists who might pursue sex reversal for detrimental exotic species. 


\section{REFERENCES}

[1] S. Abdelmalek and S. Kouachi, Proof of existence of global solutions for $m$-components reaction diffusion systems with mixed boundary conditions via the Lyapunov functional method, J. Phys. A: Math. Theory, 40, 12335-12350, 2007.

[2] N. Alikakos, $L^{p}$-bounds of solutions of reaction-diffusion equations, Commun. PDE., 4, 827$828,1979$.

[3] N.J. Bax and R.E. Thresher, Ecological, behavioral, and genetic factors influencing the recombinant control of invasive pests, Ecol. Appl., 19(4), 873-888, 2009.

[4] M.B. Bryan, D. Zalinski, K.B. Filcek, S. Libants, W. Li, and K.T. Scribner, Patterns of invasion and colonization of the sea lamprey in North America as revealed by microsatellite genotypes, Mol. Eco., 14(12), 3757-3773, 2005.

[5] J. Ding and B. Guo, Blow-up and global existence for nonlinear parabolic equations with Neumann boundary conditions, Comput. Math. Appl., 60, 670-679, 2010.

[6] A. Friedman, Partial Differential Equations of Parabolic Type, Prentice Hall Englewood Chiffs. N.J., 1964.

[7] S. Gottlieb and C.W. Shu, Total variation diminishing Runge-Kutta schemes, Amer. Math. Soc., Math. Comput., 67(221), 73-85, 1998.

[8] J.B. Gutierrez, Mathematical Analysis of the use of Trojan Sex Chromosomes as Means of Eradication of Invasive Species, PhD thesis, Florida State University, Tallahassee, FL, 2009.

[9] J.B. Gutierrez and J.L. Teem, A model describing the effect of sex-reversed $Y Y$ fish in an established wild population: The use of a Trojan Y chromosome to cause extinction of an introduced exotic species, J. Theo. Bio., 241(22), 333-341, 2006.

[10] J.E. Hill and C.E. Cichra, Eradication of a reproducing population of convict cichlids, cichlasoma nigrofasciatum (cichlidae) in north-central Florida, Florida Scientist, 68, 65-74, 2005.

[11] W.B. Fitzgibbon, S.L. Hollis, and J.J. Morgan, Stability and Lyapunov functions for reactiondiffusion systems, SIAM J. Math. Anal., 28(3), 595-610, 1997.

[12] W.D. Hamilton, Extraordinary sex ratios: A sex-ratio theory for sex linkage and inbreeding has new implications in cytogenetics and entomology, Science, 156(774), 477-488, 1967.

[13] A. Haraux and A. Youkana, On a result of K. Masuda concerning reaction-diffusion equations, Tôhoku. Math. J., 40, 159-163, 1988.

[14] D. Henry, Geometric Theory of Semi-linear Parabolic Equations, Lecture Notes in Mathematics, Springer-Verlag, New-York, 840, 1984.

[15] S.L. Hollis, On the question of global existence for reaction-diffusion systems with mixed boundary conditions, Quarterly of Appl. Math. LI, 2, 241-250, 1993.

[16] S.L. Hollis, R.H. Martin, and M. Pierre, Global existence and boundedness in reaction diffusion systems, SIAM. J. Math. Anal., 18(3), 744-761, 1987.

[17] G.A. Hunter and E.M. Donaldson, Hormonal sex control and its application to fish culture, W.S. Hoar, D.J. Randall, and E.M. Donaldson (eds.), Reproduction: Behavior and Fertility Control, Fish Physiology, Academic Press, New York, NY, 9B, 223-303, 1983.

[18] M.A. Hurley, P. Matthiesen, and A.D. Pickering, A model for environmental sex reversal in fish, J. Theo. Bio., 227(2), 159-165, 2004.

[19] I. Katsiadakiand, A.P. Scott, M.R. Hurstand, P. Matthiessen, and I. Mayer, Detection of environmental androgens: A novel method based on enzyme-linked immunosorbent assay of spiggin, the stickleback (Gasterosteus aculeatus) glue protein, Environ. Tox. Chem., 21(9), 1946-1954, 2002.

[20] E.F. Knipling, Possibilities of insect control or eradication through the use of sexually sterile males, J. Econ. Entomol, 48(4), 459-462, 1955.

[21] M. Kirane and S. Kouachi, Asymptotic behavior for a system describing epidemics with migration and spatial spread of infection, Dyn. Sys. Appl., 12, 121-130, 1993.

[22] M. Kirane and S. Kouachi, A strongly nonlinear reaction diffusion model for a deterministic diffusive epidemic, J. Indust. Appl. Math., 12(1), 29-36, 1995.

[23] M. Kirane and S. Kouachi, Global Solutions to a System of Strongly Coupled Reaction-Diffusion Equations, Nonlinear Analysis Theory, Meth. Appl., 126, 1996.

[24] S. Kouachi and A. Youkana, Global existence for a class of reaction-diffusion systems, Bull. Polish Acad. Sci., 49(3), 2001.

[25] S. Kouachi, Global existence for coupled reaction diffusion systems modelling some reversible chemical reactions, Dyn. Part. Diff. Equ., 8(2), 79-88, 2011.

[26] S. Kouachi, Existence of global solutions to reaction-diffusion systems via a Lyapunov functional, Electron. J. Diff. Equ., 68, 1-10, 2001. 
[27] S. Kouachi, Existence of global solutions to reaction-diffusion systems with nonhomogeneous boundary conditions via a Lyapunov functional, Electron. J. Diff. Eqs., 88, 1-13, 2002.

[28] J.J. Nagler, J. Bouma, G.H. Thorgaard, and D.D. Dauble, High incidence of a male-specific genetic marker in phenotypic female Chinook salmon from the Columbia river, Environ. Health Persp., 109(1), 67-69, 2001.

[29] J.H. Myers, D. Simberloff, A.M. Kuris, and J.R. Carey, Eradication revisited: Dealing with exotic species, Trends in Ecology \& Evolution, 15, 316-320, 2000.

[30] O. T. A., Harmful non-indigenous species in the United States, OTA-F-565 U.S. Congress, Office of Technology Assessment, Washington, DC, 1993.

[31] P. Palace, R.E. Evans, K. Wautierand, L. Vandenbyllardt, W.W. Vandersteen, and K. Kidd, Induction of vitellogenin and histological effects in wild fathead minnows from a lake experimentally treated with the synthetic estrogen, Ethynyl Estradiol, Water Quality Research Journal Canada, 37(3), 637-650, 2002.

[32] G.H. Rodda, C.S. Jarnevich, and R.N. Reed, What parts of the US mainland are climatically suitable for invasive alien pythons spreading from Everglades National Park? Mol. Eco., 14(12), 3757-3773, 2005.

[33] K. Masuda, On the global existence and asymptotic behavior of solutions of reaction-diffusion equations, Hokkaido. Math. J., 12, 360-370, 1983.

[34] J. Morgan, Global existence for semilinear parabolic systems, SIAM J. Math. Anal., 20, 11281144, 1989.

[35] R.D. Parshad and J.B. Gutierrez, On the existence of global attractor for the Trojan Y Chromosome model, Commun. Pure Appl. Anal., 10(1), 339-359, 2011.

[36] R.D. Parshad and J.B. Gutierrez, On the well posedness of the Trojan Y Chromosome model, Bound. Val. Prob., 1-29, 2010.

[37] A. Pazy, Semigroups of Linear Operators and Applications to Partial Differential Equations, Appl. Math. Sci., Springer-Verlag, New York, 44, 1983.

[38] B. Philips and R. Shine, Adapting to an invasive species: Toxic cane toads induce morphological change in Australian snakes, Proc. Nat. Acad. Sci. USA, 101(49), 17150-17155, 2004.

[39] M. Pierre, Global existence in reaction-diffusion systems with control of mass: A survey, Milan J. Math., 78(2), 417-455, 2010.

[40] F. Rothe, Global Solutions of Reaction-Diffusion Systems, Lecture Notes in Math., SpringerVerlag, Berlin, 1072, 1984.

[41] P.L. Shafland and K.L. Foote, A reproducing population of Serrasalmus humeralis Valenciennes in southern Florida, Florida Scientist, 42, 206-214, 1979.

[42] J. Smoller, Shock Waves and Reaction-Diffusion Equations, Springer-Verlag, New York, 1983.

[43] G. Sell and Y. You, Dynamics of Evolutionary Equations, Second Edition, Appl. Math. Sci., Springer, New York, 143, 2002.

[44] R. Temam, Infinite-dimensional Dynamical Systems in Mechanics and Physics, Springer, New York, NY, 1998.

[45] M.B. Twohey, J.W. Heinrich, J.G. Seelye, K.T. Fredricks, R.A. Bergstedt, C.A. Kaye, R.J. Scholefeld, R.B. McDonald, and G.C. Christie, The sterile-male-release technique in Great Lakes sea lamprey management, J. Great Lakes Res., 29(1), 410-423, 2003.

[46] Y. You, Global dynamics of the brusselator, Dyn. Part. Diff. Equ., 4(2), 167-196, 2007.

[47] Y. You, Asymptotical dynamics of Selkov equations, Disc. Cont. Dyn. Sys. Series S, 2, 193-219, 2009.

[48] Y. You, Global dynamics of an autocatalytic reaction diffusion system with functional response, J. Appl. Anal. Comput., 1(1), 121-142, 2011. 